Hydroécol. Appl. (1990) 1/2, pp. 135 - 149

\title{
Essai de discrimination de deux populations de vandoise (Leuciscus leuciscus, L., 1758) par étude de la forme de leurs écailles : aspects méthodologiques.
}

\section{Luc Fraisse}

CEMAGREF DQEPP, Laboratoire d'Hydroécologie Quantitative, 3, Quai Chauveau, 69336 Lyon Cedex 09.

Résumé. - Le Centre de Production Nucléaire (CPN) du Bugey rejette ses eaux de refroidissement dans le Rhône en rive droite. L'échauffement artificiel des eaux du Rhône est à i'origine d'importantes variations d'abondance des poissons échantillonnés à proximité du rejet.

Les échanges entre les populations de poissons du Rhône et de l'Ain, affluent qui débouche en rive droite à 10 kilomètres à l'aval du rejet d'eau échauffée, semblent jouer un rôle déterminant dans la stratégie adaptative des espèces présentes.

L'importance de ces échanges nécessite une caractérisation des populations présentes qui peut être obtenue par l'analyse de la forme du contour des écailles. La présente étude expose la méthode utilisée qui est appliquée à deux échantillons de vandoises (Leuciscus leuciscus, L., 1758), prélevées dans le Rhône à l'amont du CPN et dans le bassin versant de l'Ain.

La technique de saisie automatique du contour de chaque écaille, son codage, le calcul de descripteurs indépendants de l'orientation et de la taille des écailles sont brièvement présentés. L'analyse discriminante entre les deux échantillons, totalisant un effectif de 127 individus, sélectionne dix descripteurs de forme dont neuf sont constitués par les paramètres du développement en séries de Fourier du contour des écailles.

Une validation du type "jacknife" permet d'apprécier le pouvoir prédictif de cette méthode : $85 \%$ des individus sont bien affectés par l'analyse discriminante à la définition de laquelle ils n'ont pas participé. Des propositions sont formulées pour améliorer ce résultat.

Un premier examen des vandoises capturées sur le Rhône à l'aval du CPN permet de confirmer l'hypothèse de la provenance en proportion importante de vandoises de l'Ain dans la zone soumise à l'échauffement au début du printemps.

Summary. - The Bugey Nuclear Generation Centre (NGC) discharges its cooling water into the right bank of the Rhône river. Artificial heating of the Rhône water induces substantial variations in the fish count in the vicinity of the outfall. 
Exchanges of fish population from the Rhône and Ain river, tributary which flows into the right bank of the Rhone downstream of the heated water outfall, plays a leading role in the adaptative strategy of the species.

Owing to the magnitude of these exchanges, the species must be identified by analyzing the shape of the contour of their scales. The present article shows the method followed and applied to two samples of dace (Leuciscus leuciscus, L., 1758) withdrawn from the Rhône upstream of the NGC and in the catchment basin of the Ain.

The paper also gives a short description of the automatic acquisition technique of the contour of each scale, its coding, as wells as the calculation of independent descriptors of the orientation and size of scales. The discriminant analysis between two samples totalling 127 fish allows ten shape descriptors to be selected, nine of which consist of the development parameters in Fourier series of the contour of scales.

A jacknife-type validation is used to appraise the predictive power of this method tested on a sample of fish that did note participate to the discriminant analysis. $85 \%$ of the fish are indeed correctly classified by discriminant analysis. Proposal have been made to improve this result.

A first examination of dace caught in the Rhône downstream of the NGC confirms the assumption of large quantities of dace originating from the Ain river in the heated zone at the beginning of spring.

\section{INTRODUCTION}

Les études de surveillance hydrobiologique menées depuis dix ans sur le site du centre de production nucléaire du Bugey ont permis de mettre en évidence l'influence du réchauffement artificiel des eaux sur le peuplement de poissons.

D'importantes variations d'abondance sont observées dans les zones les plus directement soumises à l'élévation thermique. Les biomasses de poissons capturés deviennent faibles à partir de $24^{\circ} \mathrm{C}$ et pratiquement nulles à partir de $26^{\circ} \mathrm{C}$.

II est apparu intéressant de mieux cerner les mouvements des poissons à l'origine de la désertification de ce secteur en été, et de sa recolonisation en automne, hiver et printemps.

Dans un premier temps, il a été décidé de porter une attention toute par- ticulière à l'importance des échanges entre populations du Rhône et de l'Ain, ce dernier cours d'eau paraissant jouer un rôle de zone refuge lorsque la rive droite du Rhône connaît une température excessive.

\section{CONTEXTE DE L'ÉTUDE}

\subsection{Le site du Bugey}

Le centre de production nucléaire du Bugey (CPN) est installé à $35 \mathrm{~km}$ de Lyon sur la rive droite du Rhône, en amont de la confluence avec l'Ain.

La puissance totale du centre s'élève à $4190 \mathrm{MW}$ pour un débit prélevé de $118 \mathrm{~m}^{3} / \mathrm{s}$.

Le fonctionnement du centre de production induit une modification importante des conditions thermiques. 
Les profils thermiques réalisés par Electricité de France montrent que le mélange des eaux entre la veine d'eau chaude issue du centre et le Rhône ne s'effectue qu'après la confluence de l'Ain, pour des débits compris entre $368 \mathrm{~m}^{3} / \mathrm{s}$ et $820 \mathrm{~m}^{3} / \mathrm{s}$.

\subsection{Perspective de l'étude}

Les principales conclusions des études de surveillance hydrobiologique du site du Bugey ont été établies à partir de l'examen de la composition des échantillons de poissons récoltés sur huit stations de pêche réparties sur environ dix kilomètres de part et d'autres du centre (figure 1):
- Une différence significative apparait entre la structure des échantillons capturés à l'amont du centre et ceux provenant de la rive droite du Rhône à l'aval du rejet d'eau chaude, jusqu'au confluent de l'Ain. Les espèces dont l'abondance relative est en majeure partie à l'origine de cet écart sont la vandoise (Leuciscus leuciscus (L., 1758)) et l'ablette (Alburnus alburnus (L., 1758)).

- La composition des échantillons capturés par pêches électriques standardisées sur la station située en rive gauche du Rhône en amont du pont de Loyettes (station 6G), est proche de celle des lots prélevés à l'amont du CPN (stations 1, 2, 3); ce qui s'explique par le faible écart ther-

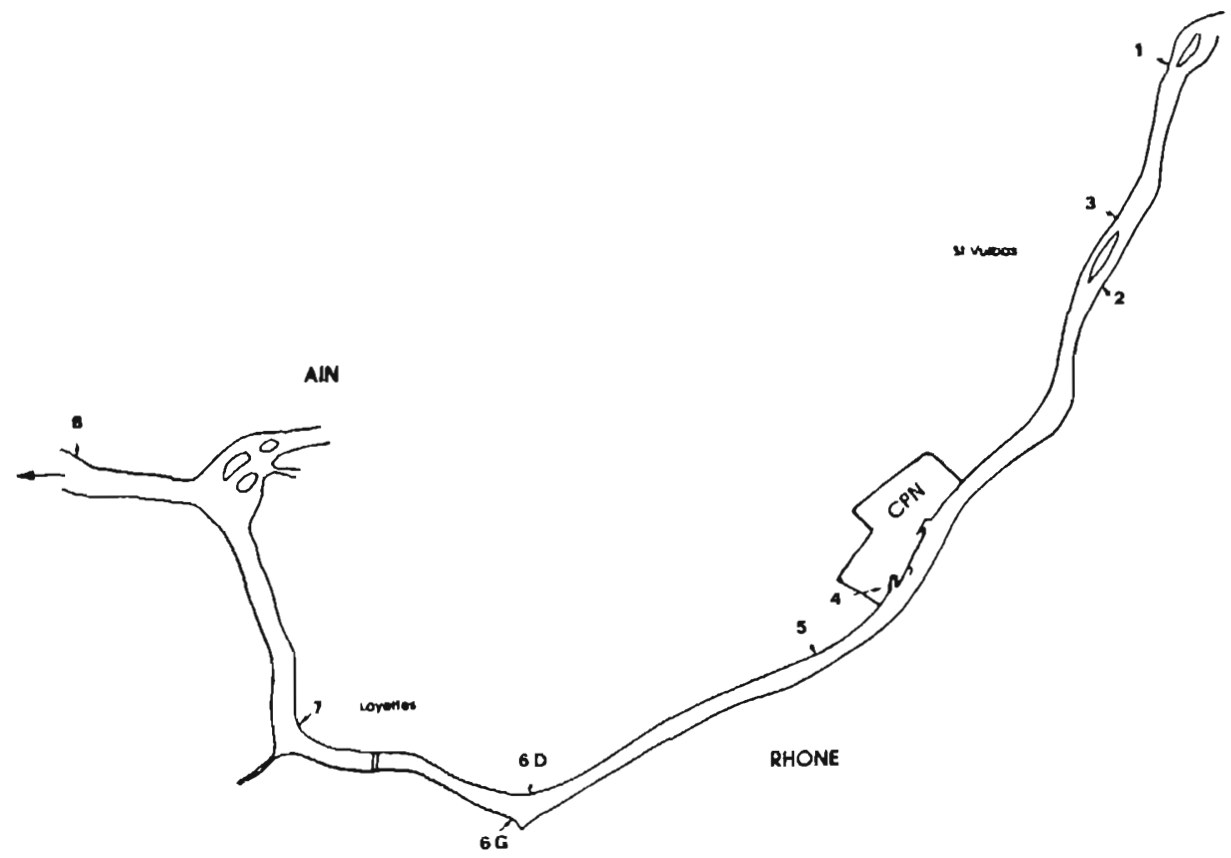

Fig. 1 - Stations d'études du suivi hydrobiologique du CPN du Bugey sur le Rhône. 
mique induit sur cette rive par le rejet d'eau chaude.

- La biomasse totale des poissons capturés sur les stations réchauffées diminue fortement en été, alors que celle obtenue sur la station située sur le Rhône à l'aval immédiat du confluent de l'Ain présente une augmentation notable.

Ces différents éléments ont conduit à étudier le rôle de l'Ain dans la recolonisation de la rive échauffée du Rhône par une espèce comme la vandoise, qui subit de façon significative les effets des élévations artificielles de température du Rhône. Cette influence est visible sur la figure 2, qui représente l'évolution de la biomasse des vandoises capturées en fonction de la date des campagnes de pêche: les biomasses correspondant aux stations situées à l'aval du centre deviennent nulles chaque année pendant l'été.

Une des questions qui se pose est de savoir si les populations de vandoise de l'Ain et du Rhône sont différenciées, et si le rétablissement en automne et en hiver des stocks de vandoise de la rive du Rhône soumise au réchauffement a pour origine un recrutement à partir des populations de l'Ain.

Cette hypothèse doit être élucidée avant que le projet d'aménagement hydraulique du confluent de l'Ain soit mis en œuvre, dans la mesure où ce-

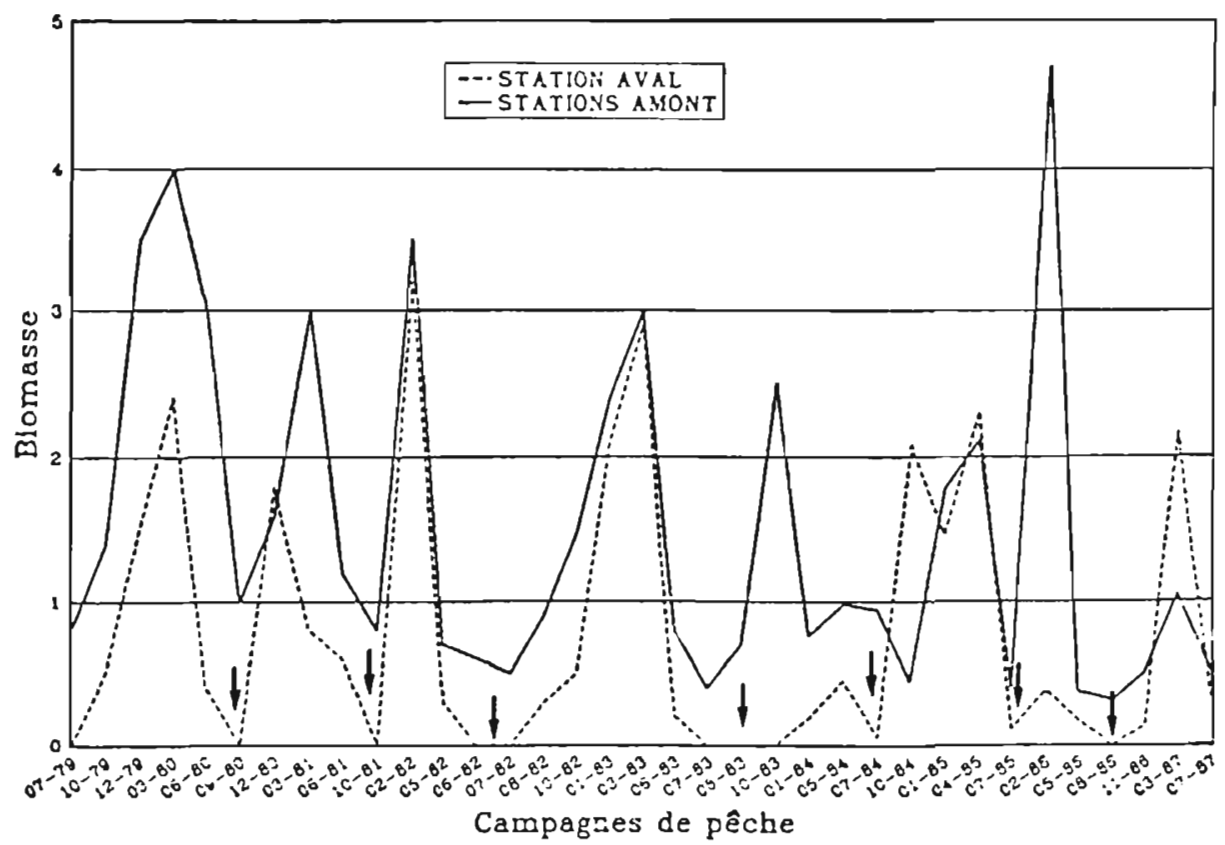

Fig. 2 - Biomasse de vandoise, exprimée en $\mathrm{kg}$ par 30 minutes de pêche électrique, pour différentes campagnes de péche. Stations amont : 1, 2, 3 sur la figure 1 ; station aval: $6 \mathrm{D}$ sur la figure 1 . 
lui-ci comporte l'édification d'un seuil qui risque de limiter notablement les échanges entre les populations de poissons des deux cours d'eau.

\section{MÉTHODES D'IDENTIFICATION}

Pour mener à bien cette étude trois méthodes d'identification des populations de vandoise seront comparées.

\subsection{La morphométrie}

Ce travail est ici réalisé par Nathalie Fabre (1989) dans le cadre d'une thèse de docteur vétérinaire, citée pour mémoire.

Deux types de caractères décrivent chaque individu.

- Caractères méristiques (dénombrement de pièces anatomiques).

- Caractères morphométriques (mensuration d'éléments du corps).

Au total 26 mesures morphométriques et méristiques ont été choisies.

Le traitement de ces données par analyse discriminante pas à pas affecte correctement les vandoises du Rhône et de l'Ain à leur groupe d'origine dans $72 \%$ des cas, en utilisant le diamètre de l'œil et le nombre de rayons de la nageoire dorsale.

\subsection{L'électrophorèse}

L'étude du polymorphisme enzymatique de certains organes du poisson par électrophorèse permet de diffé- rencier des individus appartenant à deux populations distinctes. Cette étude est menée actuellement à l'Université Lyon I par le Laboratoire de Biologie Animale.

\subsection{La scalimétrie}

Les travaux d'Hélène de Pontual sur la discrimination de stocks de saumon atlantique (Salmo salar L., 1758) par étude de la forme de leurs écailles ont permis de discriminer de façon très satisfaisante $(99 \%)$ les populations natives de différents bassins fluviaux (Pontual et Prouzet, 1988).

Cette méthode d'identification sera développée ici.

\section{DÉROULEMENT ET RÉSULTATS DE L'ÉTUDE}

\section{1 Échantillonnage}

Les campagnes de pêche électrique ont été réalisées au cours des années 1987-1988.

L'emplacement des stations où ont été pêchées les vandoises utilisées pour cette étude est présenté sur les figures 1 et 3 :

- Deux stations sont situées en amont du CPN : stations Lagnieu et Saint-Vulbas. Les individus prélevés sur ces stations sont supposés caractériser la population du Rhône.

- Les individus définissant la population de l'Ain ont été pêchés sur un 


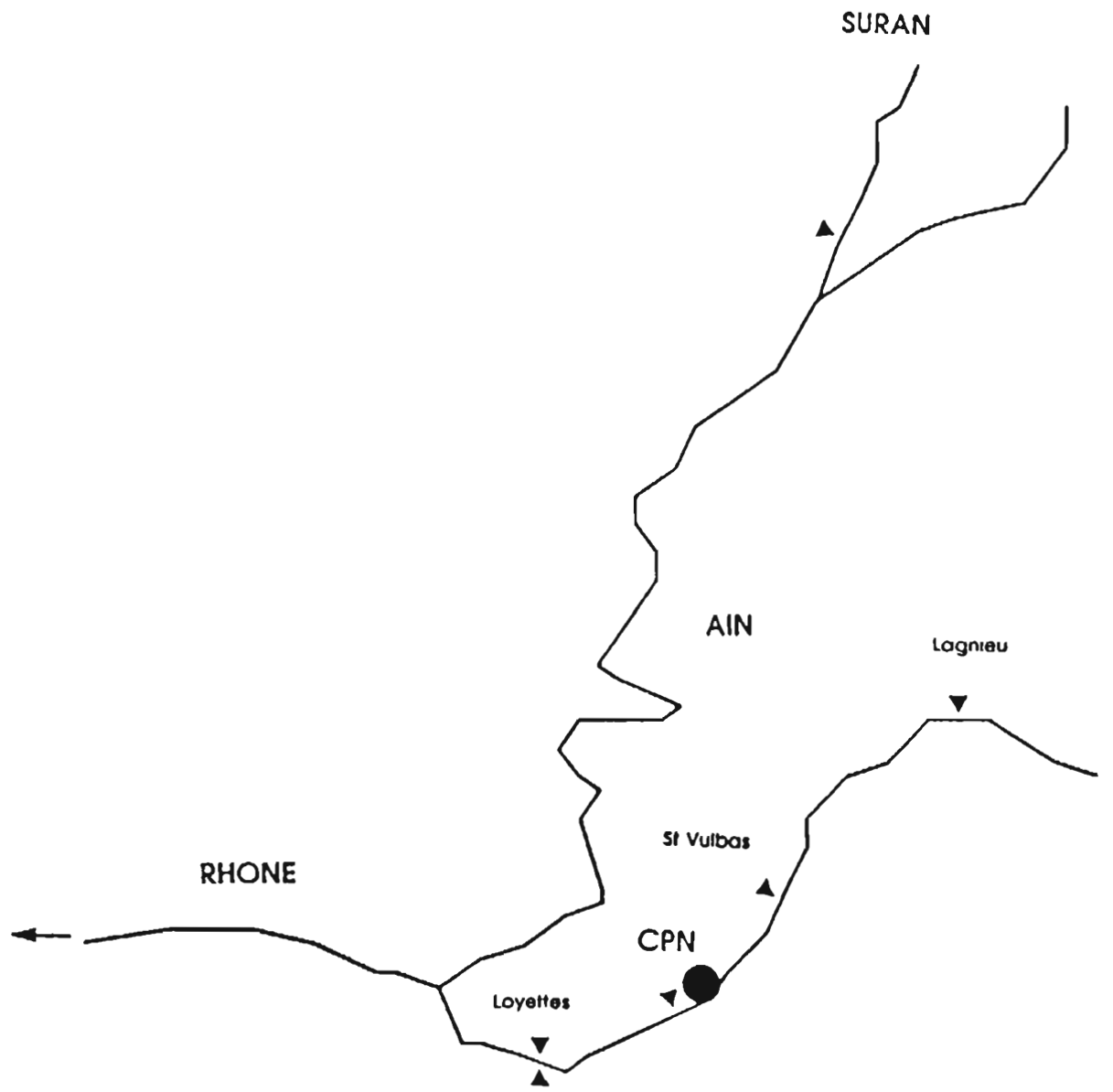

Fig. 3 : Stations de prélèvement des vandoises.

affluent de celui-ci, le Suran (à $10 \mathrm{~km}$ environ de la confluence).

- Trois stations de pêche situées à l'aval du CPN définissent les individus dits "anonymes" dont l'origine doit être étudiée :

- une station à l'aval proche du $\mathrm{CPN}$, en rive droite (5),

- une station au niveau de Loyettes en rive droite (6D),
- une station en rive gauche (6G), peu influencée par l'échauffement artificiel.

Les différentes campagnes de pêche ont permis d'obtenir :

73 individus de l'Ain,

54 individus du Rhône,

56 individus "anonymes".

Pour chaque poisson sont prélevées au minimum cinq écailles. Elles 
sont colorées à la nécrosine et montées entre deux lames de verre.

L'aire de prélèvement se situe audessus de la ligne latérale au niveau de la nageoire dorsale (fig. 4). Les écailles régénérées sont éliminées.

\subsection{Traitement d'image}

L'étude de la forme des écailles passe par l'extraction de l'information du contour et implique l'utilisation d'images numériques.

\section{a. Analyse d'image (fig. 5).}

Le système d'analyse d'image utilisé se compose d'un microscope monté sur une caméra, elle-même couplée à un processeur analogique/numérique. L'ensemble du système est piloté par un microordinateur.

La caméra vidéo transmet l'image au microscope sous la forme de signaux analogiques. Ces signaux sont ensuite numérisés en points images. Chaque pixel est caractérisé par une valeur de niveau de gris (variant de 0 à 255). La coloration de l'écaille augmente le contraste fond-objet et permet de délimiter facilement la zone interne de l'écaille.

b. Algorithmes de traitement (fig. 6).

\section{Prètraitements :}

Le prétraitement s'effectue sur l'image en niveaux de gris de taille 512768 pixels.

Un filtrage et une division d'image permettent d'uniformiser le fond par rapport à l'objet en augmentant le contraste.
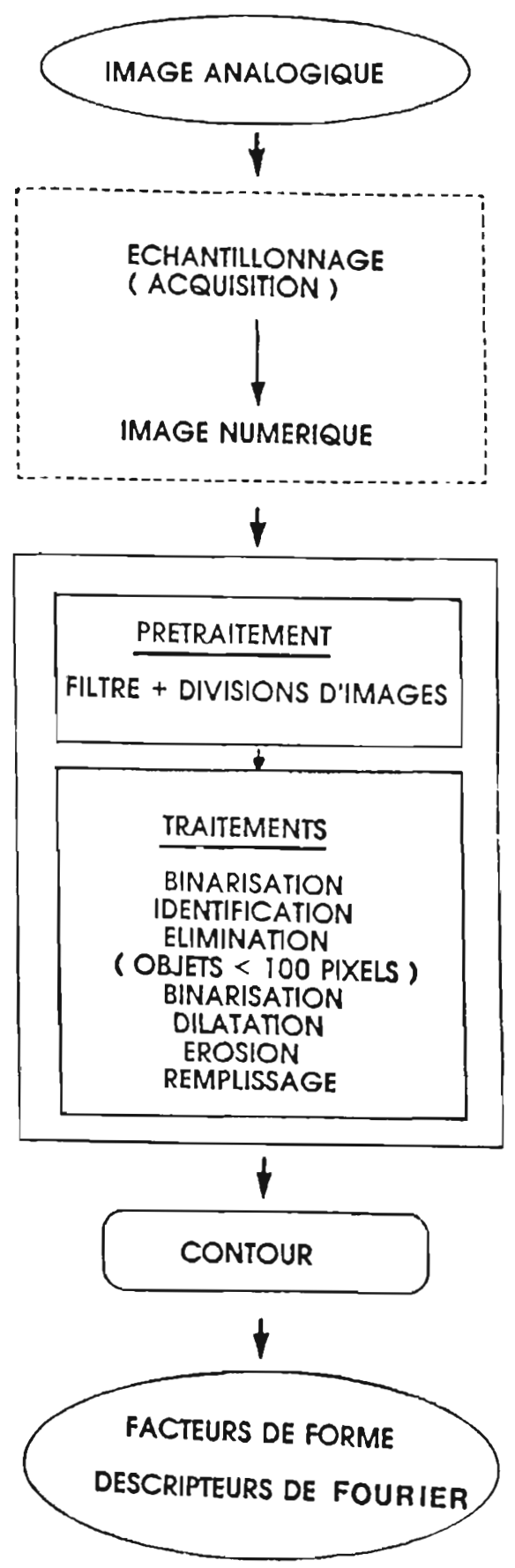

Fig. 6 : Algorithmes de traitement. 


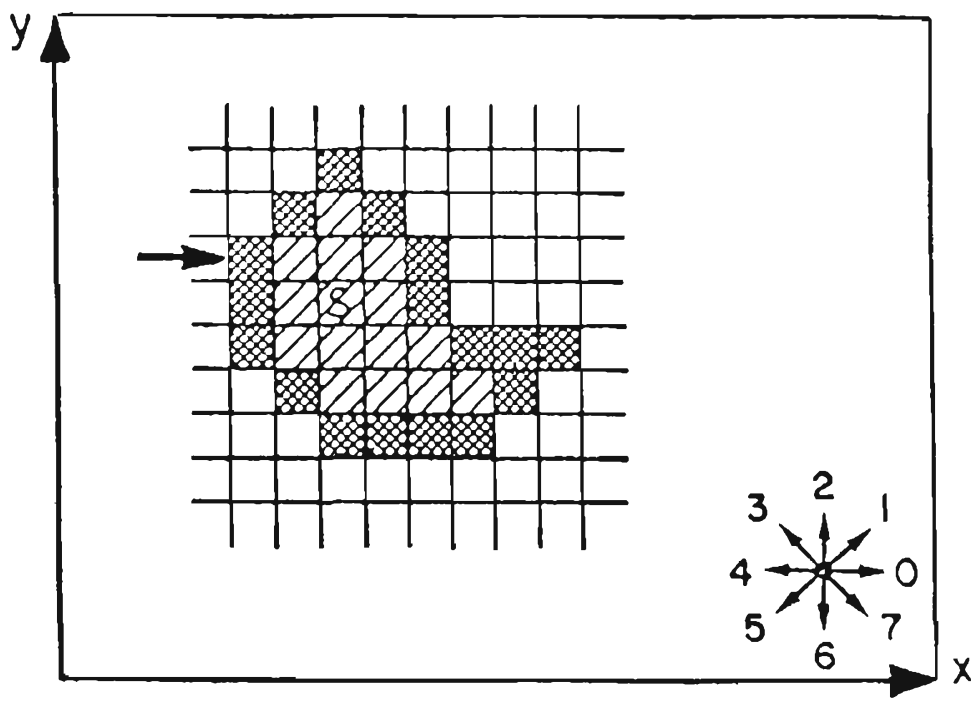

Fig. 7 : Code de Freeman (en bas à droite) et exemple de codage d'un contour. La chaîne codée est décrite à partir d'un pixel fléché et dans le sens trigonométrique : 6677000114432 335 5. D'après H. de Pontual (1988).

\section{Traitements :}

Un découpage de l'image obtenu selon deux niveaux de gris (seuillage) donne une image binaire qui caractérise l'objet, ici l'écaille), par rapport au fond. Les objets parasites sont éliminés et l'écaille est correctement reconstituée en fermant son contour par dilatation et érosion (1).

\section{Extraction du contour :}

Le stockage de la forme du contour est réalisé par la méthode du codage de Freeman (1974), qui identifie par un chiffre la direction d'un pixel à son voisin immédiat sur le contour de l'objet examiné, en se déplaçant dans le

(1) En appelant "noir" et "blanc" les caractéristiques des points après binarisation de l'image, la dilatation consiste à noircir chaque pixel voisin par un coin, d'un pixel noir. L'érosion, opération duale de la dilatation, blanchit chaque pixel voisin par un coin, d'un pixel blanc. sens contraire des aiguilles d'une montre (fig. 7).

Le contour sera donc défini par une suite de chiffres variant de 0 à 7 et représentant des directions.

\subsection{Descripteurs de forme.}

A partir du contour ainsi codé, deux types d'attributs de forme sont calculés. Ils sont indépendants de la taille et de l'orientation de l'écaille.

- Facteurs de forme.

Ils caractérisent l'élongation et la compaction de l'objet.

Six facteurs de formes sont calculés:

$\mathrm{F} 1=$ Périmètre $/ \sqrt{\text { surface }}$

$\mathrm{F} 2$ = Périmètre/longueur

$\mathrm{F} 3=$ Périmètre/largeur. 
$\mathrm{F} 4=\sqrt{\text { surface }} /$ longueur.

$F 5=\sqrt{\text { surface }} /$ largeur.

F6 = largeur/longueur.

- Cœefficients de Fourier.

Une forme plane peut être décrite par décomposition de son contour en séries de Fourier. Le contour fermé de l'écaille est approximé par deux séries:

$$
\begin{aligned}
& X(t)=A o+\sum_{1}^{n} X n(t) \\
& Y(t)=C o+\sum_{1}^{n} Y n(t)
\end{aligned}
$$

$\operatorname{avec} X n(t)=\operatorname{an}{ }^{*} \cos (2 n \pi t) / T$

$$
+b n^{*} \sin (2 n \pi t) / T
$$$$
Y n(t)=c n^{*} \cos (2 n \pi t) / T
$$

$$
+d n^{*} \sin (2 n \pi t) / T
$$

$X(t)$ et $Y(t)$ représentent les coordonnées du contour, t la distance parcourue sur le contour à partir d'un point origine, $T$ le périmètre du contour.

Kuhl et Giardina (1977) montrent que la courbe représentée par les équations paramétriques $X=X n(t)$ et $Y=Y n(t)$ est une ellipse. Un contour est donc défini par une série de $n$ ellipses ( $n=$ nombre d'harmoniques).

A partir des paramètres an, bn, $\mathrm{cn}$, $\mathrm{dn}$, sont calculés des cœfficients indépendants de l'orientation et de la taille de l'écaille :

A2...An, B1...Bn représentent la longueur du grand et du petit axe de l'ellipse correspondante, divisée par la longueur du grand axe de la première ellipse.
O2...On représentent l'angle entre un axe de référence et le grand axe de l'ellipse $n$.

P1...Pn sont les angles de déphasage, représentant la position sur l'ellipse $\mathrm{n}$ du point correspondant à l'origine de la lecture du contour, prise par définition au niveau du sommet de l'axe de référence.

L'axe de référence généralement choisi est le grand axe de la première ellipse. Dans le cas présent, celle-ci étant pratiquement assimilable à un cercle, nous avons retenu comme axe de référence celui joignant le centre de gravité de l'écaille avec le point du contour situé à égale distance des deux pointes de la partie antérieure de l'écaille.

La reconstitution du contour est d'autant meilleure que le nombre d'harmoniques $(n)$ retenu est grand, chaque nouvelle harmonique correspond à 4 paramètres supplémentaires calés de façon à réduire l'écart entre le contour réel et le contour recalculé.

Dans cette étude, dix harmoniques sont calculées pour définir chacune des écailles, la reconstitution du contour paraissant satisfaisante à ce niveau (fig. 8).

\subsection{Traitement des données. Discrimination des groupes}

Au total 43 coefficients caractérisent chaque individu: six facteurs de forme et 37 coefficients correspondant aux dix premières harmoni- 

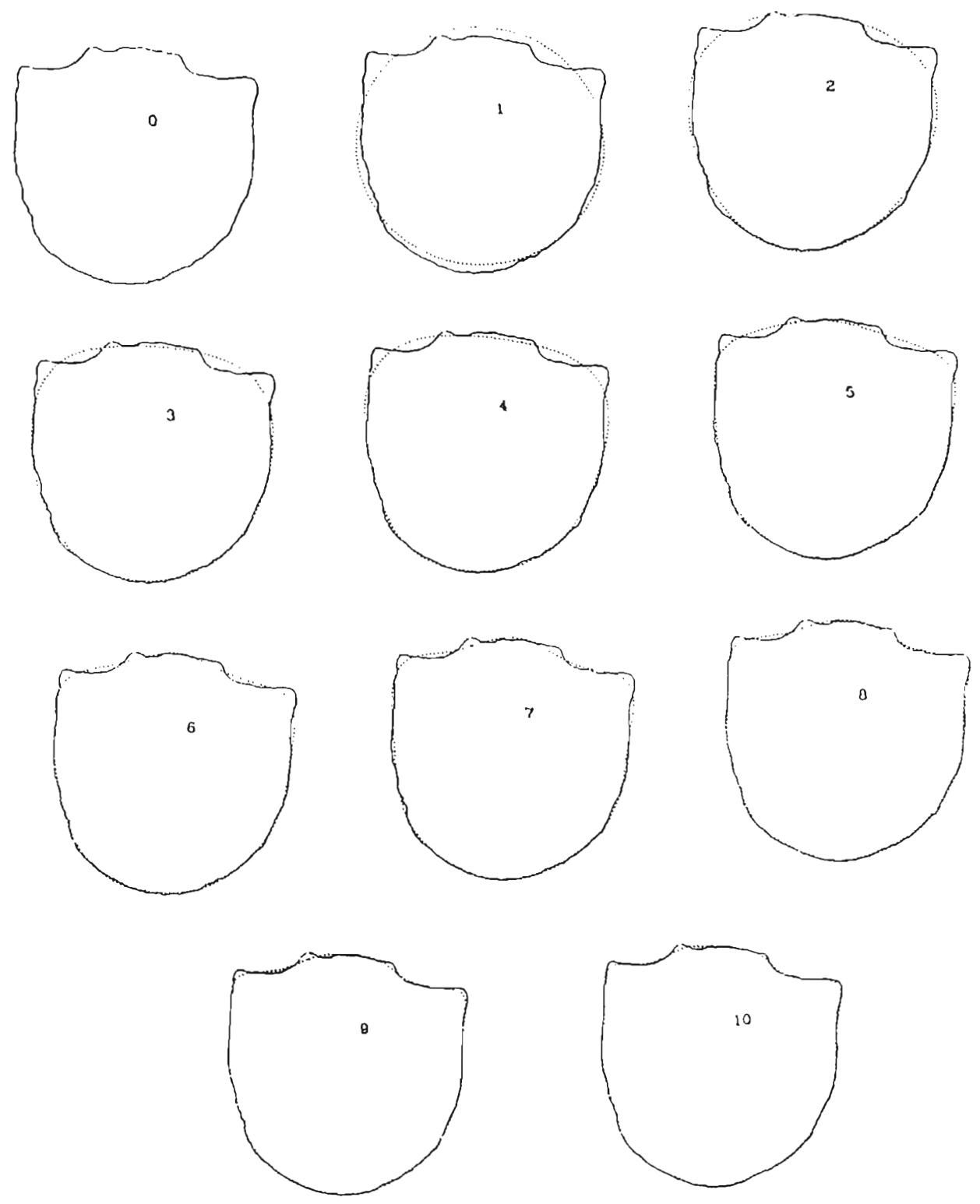

Fig. 8: Reconstitution du contour pour chaque harmonique. Le contour de l'écaille est en trait continu, le contour reconstitué est en trait pointillé. 
ques de la décomposition en séries de Fourier.
F1...F6.
A2 ....A10.
B1....B10.
O2....O10.
P2....P10.

Les premiers traitements consistent à sélectionner les variables permettant de discriminer au mieux deux groupes de vandoise: le groupe du Rhône (amont du centre) et le groupe du Suran.

\section{a. Régression multiple linéaire.}

La régression multiple permet de sélectionner un sous-ensemble de variables qui interviennent de façon significative dans la discrimination entre groupes.

La pertinence d'une variable sur la variable à expliquer est donnée par le test de Fisher, fondé sur l'analyse de la part de variance expliquée par la variable introduite par rapport à la variance résiduelle (fig. 9).
Le sous-ensemble sélectionné est le suivant :

$$
\begin{aligned}
& B(2) A(9) O(3) B(1) A(8) F 5 O(7) \\
& P(3) O(8) P(2)
\end{aligned}
$$

Deux remarques peuvent être formulées à partir de ces résultats :

- un seul facteur de forme (F5) est sélectionné,

- 9 harmoniques sont utilisées pour expliquer l'appartenance à l'un des deux groupes.

\section{b. Analyse discriminante.}

Le sous-ensemble de variables ainsi sélectionné est traité par analyse discriminante pas à pas. L'objectif est de déterminer la fiabilité de l'affectation d'un individu à un groupe.

Le pourcentage de bien classés est calculé pour chaque introduction de variable. Les résultats sont présentés sur la figure 10.

Au dixième pas, le pourcentage d'individus bien classés est de $88 \%$.

P de Fisber

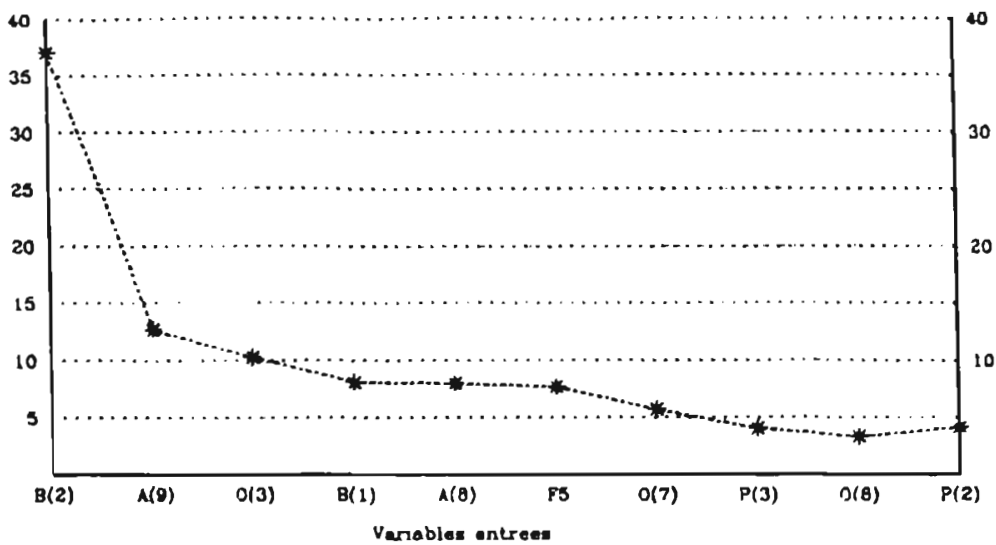

Flg. 9 : Régression Stepwise. 


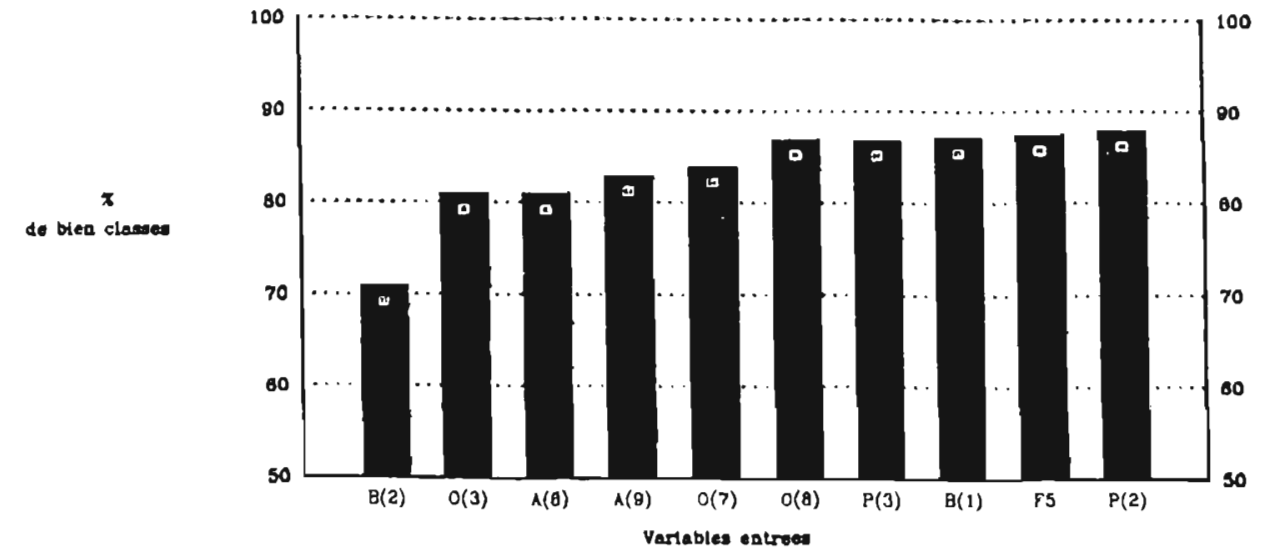

Fig. 10 : Analyse discriminante pas à pas.

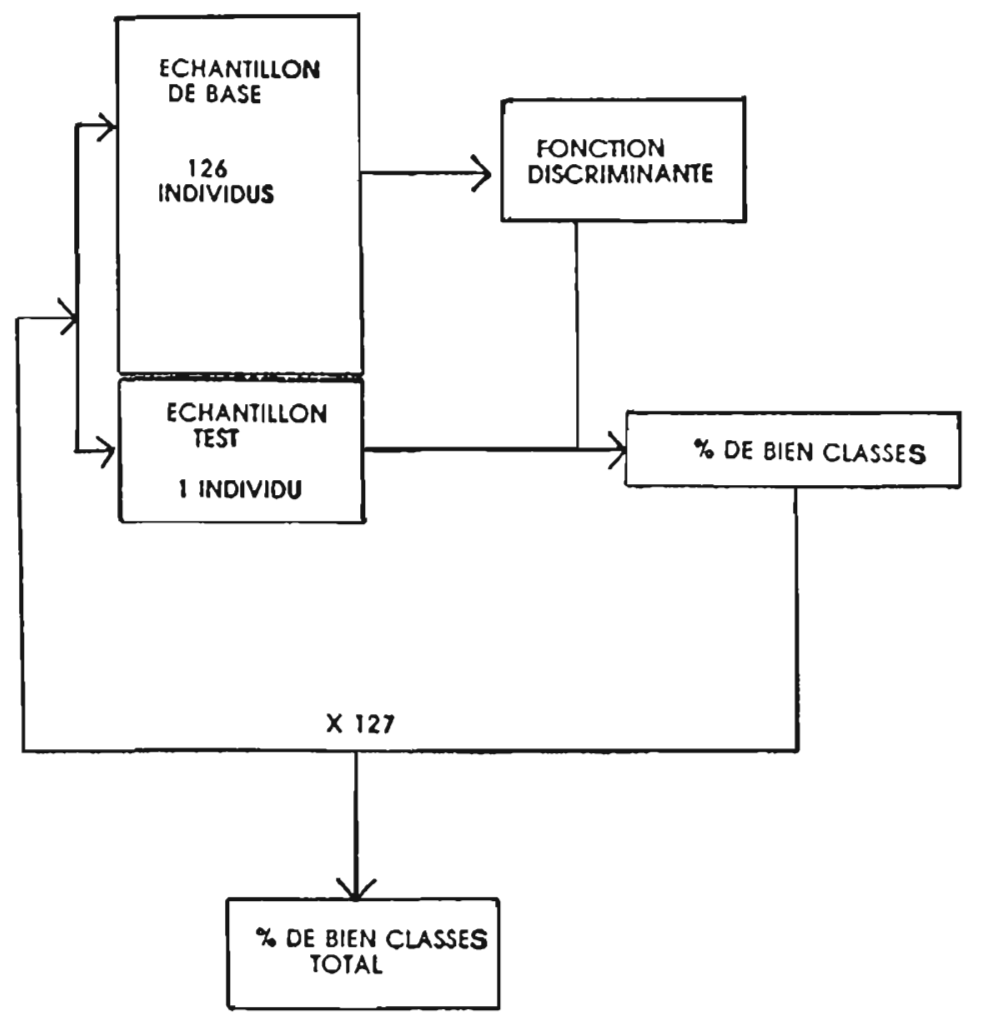

Fig. 11 : Schéma de la méthode Jacknife. 


\section{c. Test de l'analyse.}

Les résultats obtenus sont testés par la méthode dite "Jacknife" (fig. 11).

La fonction discriminante est calculée sur un échantillon de base constitué par l'ensemble des individus sauf un qui est utilisé comme individu test.

Chaque individu est utilisé à son tour comme individu test. Son affectation est effectuée par une analyse discriminante indépendante de ses caractéristiques.

Cent-vingt-sept analyses discriminantes sont donc effectuées.

On obtient donc un pourcentage de bien classés total caractérisant la valeur prédictive de l'analyse discriminante.

Un pourcentage de $85 \%$ d'individus bien classés avec les dix variables sélectionnées permet de conclure à une bonne valeur prédictive de la méthode utilisée.

\section{5 Étude des individus anonymes}

Pour les vandoises du Rhône capturées à l'aval du centre, l'application de l'analyse discriminante avec dix variables caractéristiques de la forme des écailles donne les résultats suivants :

$61 \%$ des poissons pêchés en rive droite à l'aval immédiat du CPN (Station 5 , figure 1) en mars sont affectés au groupe du Suran.

$86 \%$ des poissons capturés en septembre en rive gauche du Rhône au niveau de Loyettes (station 6G en zone peu ou non échauffée), sont affectés au groupe du Rhône.

\section{DISCUSSION}

La méthode utilisée pour discriminer deux populations de vandoise à partir de la caractérisation de la forme de leurs écailles paraît satisfaisante.

Elle présente le grand avantage, par rapport aux mesures biométriques classiques, d'être indépendante de l'opérateur. Ceci exige toutefois de définir avec précision une aire de prélèvement d'écailles, ce qui n'a pas été effectué au début du présent travail. Les moins bons résultats enregistrés ici ( $85 \%$ d'individus bien classés) par rapport à ceux obtenus par $H$. de Pontual (1988) sur le saumon atlantique (99\% d'individus bien classés) peuvent s'expliquer en partie par cette omission.

La comparaison entre les résultats obtenus par cette analyse et ceux donnés par l'électrophorèse permettra sans doute de mieux comprendre la valeur de cette discrimination et de l'interpréter en termes de génétique et de dynamique des populations.

Suite à cette étape méthodologique, un échantillonnage stratifié des

Le programme d'analyse discriminante utilisé est celui de la bibliothèque diffusée par l'ADDAD (Association pour le Développement et la Diffusion de l'Analyse des Données). Il a été écrit par J.M. Romeder.

L'analyse d'images a èté réalisée sur un matériel IBAS 2000 de la Division Technologie des Equipements Agricoles et Alimentaires du CEMAGREF de Montpellier, avec l'aide de M. André Miralles. 
vandoises de l'Ain et du Rhône pourra permettre de mieux caractériser, sur le plan spatial et temporel, les échanges entre les populations des deux cours d'eau, échanges qui paraissent déjà en première analyse importants dans les processus de recolonisation de la zone du Rhône fortement influencée par le rejet des eaux chaudes du Centre de Production Nucléaire du Bugey.

\section{BIBIIOGRAPHIE}

De Pontual H. et Prouzet P., 1988. Numerical analysis of scale morphology to discriminate between atlantic salmon stocks. Aquat. Living Resour. (1), pp. 17-27.

Fabre N., 1989. Etude morphométrique et scalimétrique des stocks de vandoises du Rhône et de l'Ain. Thèse de Doctorat Vétérinaire. ENV Alfort. $120 \mathrm{p}$.

Freeman H., 1974. Computer Processing of line drawing Images. Computing surveys, 6 (1), pp. 57-97.

Kuhl F.P. et Giardina C.R., 1982. Elliptic fourier features of a closed contour. Computer graphics and image processing, 18, pp. 236-258.

Willer R.G., 1974. The Jacknife, a review. Biometrika vol. 61, pp. 1-15.

Zahl S., 1977. Jacknifing and index of diversity. Ecology (58), pp. 907-913. 